\title{
Level of Implementation Fidelity of Youth-Friendly Services in Northwest Ethiopia
}

This article was published in the following Dove Press journal:

Risk Management and Healthcare Policy

\author{
Yohannes Ayanaw Habitu' \\ Gashaw Andargie Biks (D) ${ }^{2}$ \\ Abebaw Gebeyehu Worku' \\ Kassahun Alemu Gelaye $\mathbb{D}^{3}$ \\ 'Department of Reproductive Health, \\ Institute of Public Health, College of \\ Medicine and Health Sciences, University \\ of Gondar, Gondar, Ethiopia; \\ ${ }^{2}$ Department of Health System and \\ Policy, Institute of Public Health, College \\ of Medicine and Health Sciences, \\ University of Gondar, Gondar, Ethiopia; \\ ${ }^{3}$ Department of Epidemiology and \\ Biostatistics, Institute of Public Health, \\ College of Medicine and Health Sciences, \\ University of Gondar, Gondar, Ethiopia
}

Correspondence: Yohannes Ayanaw Habitu

University of Gondar, P.O. Box: 196

Tel +25I-90I 460706

Fax +25I-58 8I2 90II

Email yohaneshabitu@gmail.com
Background: Youth-Friendly Services (YFS) is an evidence-based public health intervention aimed to reduce sexual and reproductive health problems among youth. To achieve the desired outcomes, YFS has to be implemented with a high level of fidelity. However, there is an evidence gap in its implementation with fidelity. Hence, the main objective of the study was to describe the level of implementation fidelity of YFS among public health centers in Central Gondar Zone, northwest Ethiopia. Therefore, this study investigated the level of implementation fidelity of YFS among public health centers in Central Gondar Zone, northwest Ethiopia.

Methods: An institutional-based cross-sectional study was conducted from September to December in 2019. A total of 1029 youths, who came to 11 health centers where YFS has been implemented were randomly selected. A validated tool, comprised of 65 Likert scaled items, designed to measure the three dimensions of fidelity was used to collect data. Face to face clients exit interview approach was used to collect the data. Summary statistics were calculated for each fidelity dimension first, and then the overall fidelity of implementation score was computed.

Results: Overall, only $48(4.7 \% ; 1.6-11.3 \%)$ of youth received the YFS with a high level of implementation fidelity. Whereas 770 (74.8\%; 57.6-90.5\%) and $211(20.5 \%$; 912.7-31.0\%) of youth received the YFS with a medium and low level of implementation fidelity, respectively. Considering each fidelity dimension, 241 (23.4\%) youth received the YFS with a high level of adherence, $212(20.6 \%)$ received the YFS with a high level of the structural quality of care, $323(31.4 \%)$ received the YFS with a high level of process quality of care, 508 (49.3\%) received the YFS with a high level of outcome quality of care, and 114 $(11.1 \%)$, received the YFS with a high level of participant responsiveness.

Conclusion: The identified low level of implementation fidelity of YFS indicates the urgent need to strengthen the YFS by the government and programmers. Further studies that can address barriers to the implementation fidelity of Youth-Friendly Services are recommended. In addition, further analysis to know the factors that may influence the fidelity of YFS are recommended.

Keywords: fidelity, adherence, implementation, youth-friendly services, Ethiopia

\section{Background}

Youth are people aged 15 to $24 .{ }^{1}$ Globally, there are around 1.8 billion youth, accounting for one out of six people. Of them, $87 \%$ live in developing countries. ${ }^{2-4}$ In the Ethiopian context, youth represent about $33 \%$ of the national population dividend. ${ }^{5}$ They have faced many health problems that could be due to low YouthFriendly Service (YFS) utilization, poor YFS delivery, cultural and other social determinants. $^{6-8}$ 
YFS is a promising evidence-based intervention, which was designed by the World Health Organization (WHO) that aimed to avert the Sexual and Reproductive Health (SRH) problems of youth. ${ }^{9,10}$ The WHO recommended YFS should be accessible, acceptable, equitable, appropriate and effective for youth ${ }^{11-14}$ and it has previously been highlighted as a successful model for providing SRH services integrated within a public health system. ${ }^{15,16} \mathrm{~A}$ range of services like counseling on sexuality, Human Immune Deficient Virus (HIV) counseling and testing, pregnancy testing, contraceptive provision, Sexually Transmitted Infections (STIs) screening and management, abortion care, and other medical services are integrated and provided in the YFS. ${ }^{17,18}$ In Ethiopia, YFS has been implemented in public health centers with monitoring and supervision held by Federal Ministry of Health (FMoH), since 2013. ${ }^{16}$

Currently, many youths require SRH services that can address their diverse needs. ${ }^{19-21}$ Hence, YFS was developed as a promising approach to deliver health services to meet youth's SRH need. ${ }^{9,10,17}$ Even if YFS intervention is implemented in countries like Ethiopia, ${ }^{16}$ youth suffer from many SRH problems like teenage pregnancy, fistula, abortion, and STIs including HIV. ${ }^{7,15,19,20,22}$ Low implementation fidelity of YFS could be one of the possible reasons claimed for the high prevalence of SRH problems among youth. ${ }^{23,24}$

Most previous studies focused on the prevalence of YFS utilization, ${ }^{25-27}$ barriers limited youth's access to YFS, ${ }^{28,29}$ and few on YFS quality of delivery. ${ }^{30}$ Besides, low quality of delivery ${ }^{30,31}$ and low positive experience among youth with the service ${ }^{32}$ were some of the reasons claimed for the low proportion of YFS use. Pieces of evidence showed, implementing programs with high fidelity, play a vital role in increasing client satisfaction, service utilization and to achieve the intended intervention outcomes. ${ }^{23,24,33}$ Though various international and national organizations have assisted in the expansion of YFS, there is study limitation on its level of implementation fidelity. ${ }^{15}$ Complex programs, such as YFS, in which many services are integrated and provided at a time, have faced challenges in delivering the intervention with high implementation fidelity. ${ }^{18}$

Monitoring implementation fidelity contributes to future improvements of interventions, which are necessary to increase the likelihood of reaching intended outcomes. ${ }^{23,24,34-36}$ Furthermore, studying on Fidelity of Implementation (FoI) efforts may also lead to better replications and sustainability of an intervention like YFS. ${ }^{35,37}$ Therefore, it is vital to investigate the level of implementation fidelity of YFS. ${ }^{32}$

Measuring the implementation fidelity of services like YFS are conceptually developed from three major constructs called adherence, quality of service delivery and participant responsiveness. ${ }^{23,24}$ Hence, it is vital to quantify the three main constructs that are intended to measure fidelity of YFS. ${ }^{23,24}$

There is an evidence gap on the level of implementation fidelity of YFS in developing countries including Ethiopia, specifically in the study area. The main objective of this study was to describe the level of implementation fidelity of YFS in this specific study area. To measure the overall fidelity of YFS, the three constructs used to measure the fidelity of YFS (adherence, quality of YFS delivery and participant responsiveness) were quantified separately. Therefore, this study aimed to investigate the level of implementation fidelity of YFS in public health centers in Central Gondar Zone, northwest Ethiopia. The findings of the study may help to strengthen and implement the YFS with high fidelity.

\section{Methods}

\section{Study Design and Setting}

An institution-based cross-sectional study was conducted among youth who utilized the YFS, from randomly selected public health centers (implementing the intervention), in Central Gondar Zone, northwest Ethiopia, from September to December 2019. Central Gondar Zone has a total of 15 districts, 466 kebeles (the smallest administrative units), and a total population of $2,655,844$, while youth accounting for $34.6 \%$ of the total population. There are 84 public health centers, though 35 of the HCs were implementing YFS, according to the 2018 Zonal report. ${ }^{38}$

\section{Study Population and Inclusion Criteria}

The source population of the study was youth of age 15-24 who came for YFS in the study area and the study population were youth of age 15-24 who utilized YFS in that specific area and available during at the time of the study. Youth who are available provided informed and written consent during the data collection period were included in the study; while those youth who were critically ill and unable to respond during the data collection period. 


\section{Sample-Size Determination}

To determine the sample size for this study, a pilot study was conducted from 60 youths attending YFS, from randomly selected HCs called Enfranz, Han and Bahir Dar. These three HCs were selected as they have already implementing YFS and have a similar setting with the main study area. Then, the sample size was calculated by using the single population proportion formula ${ }^{39}$ and taking the proportion of the high level of implementation fidelity of YFS $\mathrm{P}=26.7 \%$ from the pilot study, $95 \% \mathrm{CI}$, margin of error of $4 \%$, design effect of 2 , and $10 \%$ nonresponse rate. The final calculated sample size was 1034 .

\section{Sampling Procedure}

From the 15 districts in the zone, 6 were randomly selected by lottery method. Then, if there are two or more $\mathrm{HCs}$ implementing YFS in each district, $1 \mathrm{HC}$, was selected by lottery method. Finally, 11 HCs named Gondar (GHC), Azezo (AZHC), Tseda (THC), Gebriel (GEBHC), Woleka (WHC), Maraki (MHC), Amba Giorgis (AGHC), Maksegnit (MKHC), Kolladiba (KHC), Chuahit (CHHC) and Delgie (DHC) were included. Then, samples were correspondingly allocated across each $\mathrm{HC}$ to get representative participants. Thereafter, participants were selected using a systematic sampling technique during working hours of the week in each HC.

\section{Tool Development, Validation and Adaptation Processes Used to Measure the Implementation Fidelity of YFS}

An instrument comprised 17 general items; which measured the socio-demographic and other individual characteristics were used. Also, a validated scale with 65 items with 5 category Likert scale scores for assessing the implementation fidelity of YFS was employed. The 65 items scales were mainly from the WHO-Plus standard tool (quality assessment tool). ${ }^{8}$ The scale was developed to measure the three dimensions of fidelity ie, adherence, quality of service delivery, and participant responsiveness. Then, a fidelity score was developed for each fidelity domain, based on 5 Likert scale level. The scale passed the standard tool validation process, starting from face validity, content validity, construct validity, pilot tested and finally internal consistency was checked using the information from the pilot study. The overall instrument development, validation and adaptation processes are described below.

\section{Item Generation}

A comprehensive literature review was done and a pool of items having a total of 113 questions intended to measure the three constructs (adherence, quality of delivery and participant responsiveness) of implementation fidelity of YFS was generated as an initial point.

\section{Face and Content Validity}

The first round of face and content validity was determined by 6 professionals' who independently reviewed items in the questionnaire and provided ratings on the importance of each item. In addition, they rated their level of agreement for the importance of each question as very important, important, somewhat important or not important. Moreover, they qualitatively evaluated/checked on layout and readability, meaning and clarity of wording, feasibility of each item related to study participants.

In the second round of face and content validity, a panned discussion including another 6 professional experts and 2 language experts was conducted. Hence, corrections like wordings, questions were phrased in positive tone, misclassification of variables, logical order of items from all constructs, repeated questions, ambiguous and vague words and double question at a time were separated. In addition, logical arrangement, appearance, layout, acronym, jargons and technical language removed, leading questions avoided, and many editorial issues were raised and edited. Finally, the comments, corrections, suggestions, judgments and opinions of the professional and language experts were considered.

Standard translation/back-translation procedures were performed to prepare a preliminary draft of the scale in Amharic (local language). In the first stage, the original instrument was translated into Amharic by two professional language translators; and in the next stage, the Amharic version was translated into English by two other professional translators who were familiar with the main study topic. In the third stage, the back translated English version of the tool was compared with the original version in terms of content, and idiomatic and semantic affinity. Minor corrections were made as a consequence and the final draft was approved by the researchers.

\section{Item Reduction}

After the first and second rounds of expert validity, 42 items were reduced and a total of 71 items were left for the pilot study. These 42 items were reduced due to criteria 
like decisions by experts as not content valid (10 items) and duplicates (32 items). Then, the pilot study was conducted using the 71 items to cheek the cultural adaptation of the tool.

\section{Results of Internal Consistency After Conducting the Pilot Study}

After the pilot study, 6 items were reduced after checking for internal consistency using the Crombach alpha value. Finally a valid and reliable instrument having 65 items in the three constructs were developed to measure the FoI of YFS.

Finally, 9 items were developed to measure the participant responsiveness dimension, with high internal consistency (Cronbach's alpha value of 0.85), 15 items were developed to measure the adherence dimension with Cronbach's alpha value of 0.91 and 41 items were developed to measure the quality of delivery dimension, with high internal consistency having Cronbach's alpha value of 0.93 . The quality of delivery dimension was further constructed based on the Donavidian model quality of care framework, that aimed to assess the structural quality (11 items), process quality (23 items) and outcome quality dimensions ( 7 items).

\section{Data Collection Procedures}

Data were collected by an interviewer-administered, predetermined and validated questionnaire. Eleven BSc holders (5 Health Officers, 6 Midwives), who had special training on YFS and working out of the data collection area, collected the data. The data collectors were health care professionals who were working as heath care providers out of the study area. In addition, the data collectors were not involved in the implementation of the YFS intervention in any of the settings. One supervisor having a Master of public health and with work experience on supervision in research data collection was involved.

\section{Data Quality Control}

To control the data quality, three days of training was provided to 11 data collectors and one supervisor before the actual data collection. Besides, the instrument was validated, so that the face validity, content validity, construct validity, internal consistency of the instrument was found high. Moreover, appropriate modifications were made on the instrument, after conducting the pilot study. The questionnaire was translated into local language (Amharic) and backtranslated to the English by one language expert and one public health expert. Then, to ensure consistency, the
Amharic version of the instrument was back-translated into the English language by another English language expert and by another professional expert.

\section{Keeping Confidentiality}

The names and other identifiers of the respondents were not recorded in the questionnaire. The information collected in each health facility was submitted to the supervisor and the principal investigator on a daily bases. The collected data were locked in a secured shelf and nobody will be allowed to see the information (except the research team).

\section{Data Analysis}

Data were analyzed using STATA version 14. Descriptive statistics with summaries of each fidelity dimension was computed first; afterwards the overall fidelity score was computed using 3 main dimensions of fidelity. The unit of analysis was at an individual level, and then aggregated into the $\mathrm{HC}$ level with total implementation fidelity scores were computed separately into three levels. Those youth whose total fidelity score of greater than or equal to $75 \%(\geq 243.75 / 325)$ was declared as had received the YFS intervention with high Fidelity of Implementation (FoI), those youth whose total fidelity score from $50 \%$ $(162.5 / 325)$ to $75 \%(243.74 / 325)$ was declared as had received the YFS intervention with medium FoI and those youth whose total fidelity score below $50 \%$ $(<162.5 / 325)$ were declared as had received the YFS intervention with low FoI. ${ }^{8,36}$ Regarding each dimension of fidelity measure, we used similar cutoff percentages for each dimension and the results are presented in tables.

\section{Results}

\section{Socio-Demographic Characteristics}

Of the total 1034 youths, 1029 (99.5\%) responded to the survey. The majority, 717 (69.7\%), were between age 20-24, and $752(73.1 \%)$ were females. Regarding their religion, 874 (84.8\%), were Orthodox Christians, and $781(75.9 \%)$ of them were urban residents. Concerning their educational status, the majority, 453 (44.0) of them were attending secondary education, and 601 (58.4\%) were unmarried (Table 1).

\section{Youth-Friendly Services Utilization}

Regarding the YFS services, 223 (21.7\%) got contraceptive counseling, $308(29.9 \%)$ got contraceptive method 
Table I Socio-Demographic Characteristics of the Respondents, Northwest Ethiopia in 2019

\begin{tabular}{|c|c|c|}
\hline Variables & Frequency & Percentage \\
\hline \multicolumn{3}{|l|}{ Age (in years) } \\
\hline $15-16$ & 58 & 5.6 \\
\hline $17-19$ & 254 & 24.7 \\
\hline $20-24$ & 717 & 69.7 \\
\hline \multicolumn{3}{|l|}{ Sex } \\
\hline Male & 277 & 26.9 \\
\hline Female & 752 & 73.1 \\
\hline \multicolumn{3}{|l|}{ Place of residence } \\
\hline Urban & 781 & 75.9 \\
\hline Rural & 248 & 24.1 \\
\hline \multicolumn{3}{|l|}{ Religion } \\
\hline Muslim & 147 & 14.4 \\
\hline Orthodox & 874 & 84.8 \\
\hline Other Christians* & 8 & 0.8 \\
\hline \multicolumn{3}{|l|}{ Educational status } \\
\hline Unable to read and write & 58 & 5.6 \\
\hline Able to read and write & 5 & 0.5 \\
\hline Primary education (I-8) & 210 & 20.4 \\
\hline Secondary education (9-12) & 453 & 44.0 \\
\hline Vocational/Diploma & 211 & 20.5 \\
\hline Degree and above & 92 & 9.0 \\
\hline \multicolumn{3}{|l|}{ Marital status } \\
\hline Unmarried & 601 & 58.4 \\
\hline Married & 392 & 38.1 \\
\hline Divorced & 23 & 2.2 \\
\hline Partner died & 13 & 1.3 \\
\hline \multicolumn{3}{|l|}{ Living arrangement } \\
\hline Alone & 194 & 18.9 \\
\hline Others** & 53 & 5.2 \\
\hline With my families & 446 & 43.3 \\
\hline With my husband & 307 & 29.8 \\
\hline With my wife & 29 & 2.8 \\
\hline \multicolumn{3}{|l|}{ Work for money now } \\
\hline No & 593 & 57.6 \\
\hline Yes & 436 & 42.4 \\
\hline \multicolumn{3}{|l|}{ Mother alive now } \\
\hline No & 126 & 12.2 \\
\hline Yes & 903 & 87.8 \\
\hline \multicolumn{3}{|l|}{ Father alive now } \\
\hline No & 312 & 30.3 \\
\hline Yes & 717 & 69.7 \\
\hline \multicolumn{3}{|l|}{ Do you have peer friend now } \\
\hline No & 201 & 19.5 \\
\hline Yes & 828 & 80.5 \\
\hline
\end{tabular}

(Continued)
Table I (Continued).

\begin{tabular}{|l|l|l|}
\hline Variables & Frequency & Percentage \\
\hline Have you ever talked about the & & \\
importance of YFS with your peer & & \\
friend/s ( $n=828)$ & & \\
No & 248 & 30.0 \\
Yes & 580 & 70.0 \\
\hline
\end{tabular}

Notes: Others implied: *protestant, Catholic, **with my uncle, aunt, grandmother/ grandfather.

collection, 263 (25.6\%) got screening for STIs, 167 (16.2) peer counseling services, $49(6.5 \%)$ abortion services, 38 (5.0\%) post-abortion care services, $173(23.0 \%)$ pregnancy testing services, and $75(7.3 \%)$ got counseling on the risks of having sex before 18 years (Table 2).

\section{Level of Adherence to Youth-Friendly Services}

Considering the level of adherence dimension, 190 (18.5\%) youth get the YFS with a low level of adherence, $598(58.1 \%)$ with a medium and 241 (23.4\%) with a high level of adherence. Tseda HC provided the YFS with the highest level of adherence 55 (58.5\%), followed by Delgie HC, 53 (56.4\%) (Table 3).

\section{Level of Quality of Delivery of Youth-Friendly Services}

Regarding the structural quality of care dimension, 127 $(12.3 \%)$ get the YFS with a low level of the structural quality of care, $690(67.1 \%)$ get the YFS with a medium, and $212(20.6 \%)$, get the YFS with a high level of the structural quality of care. Azezo HC provided the YFS with a high level of the structural quality of care 45 (47.9\%) followed by Tseda HC 39 (41.5\%). On the process quality of care dimension, $103(10.0 \%)$, get the YFS with a low level of process quality of care, $603(58.6 \%)$, get the YFS with a medium, and 323 (31.4\%), get the YFS with a high level of process quality of care. Azezo HC provided the YFS with a high level of process quality of care 82 (87.2\%) followed by Tseda HC 63 (67.0\%). Considering the outcome quality of care, $146(14.2 \%)$ get the YFS with a low level of outcome quality of care, 375 (36.4\%) get the YFS with a medium, and $508(49.4 \%)$ get the YFS with a high level of outcome quality of care. Azezo HC provided the YFS with a high level of outcome quality $82(87.2 \%)$ followed by Delgie HC 70 (74.5\%) (Table 4). 
Table 2 Youth-Friendly Services Utilization Related Characteristics of the Respondents in Northwest Ethiopia, 2019

\begin{tabular}{|c|c|c|}
\hline Variables & Frequency & Percent \\
\hline \multicolumn{3}{|l|}{ VCT services } \\
\hline No & 579 & 56.3 \\
\hline Yes & 450 & 43.7 \\
\hline \multicolumn{3}{|l|}{ Peer counseling services } \\
\hline No & 862 & 83.8 \\
\hline Yes & 167 & 16.2 \\
\hline \multicolumn{3}{|l|}{ Pre abortion counseling services $(n=752)$} \\
\hline No & 716 & 95.2 \\
\hline Yes & 36 & 4.8 \\
\hline \multicolumn{3}{|l|}{ Abortion services $(n=752)$} \\
\hline No & 703 & 93.5 \\
\hline Yes & 49 & 6.5 \\
\hline \multicolumn{3}{|l|}{ Pregnancy test $(n=752)$} \\
\hline No & 579 & 77.0 \\
\hline Yes & 173 & 23.0 \\
\hline \multicolumn{3}{|l|}{ Counseling on nutrition } \\
\hline No & 984 & 95.6 \\
\hline Yes & 45 & 4.4 \\
\hline \multicolumn{3}{|l|}{$\begin{array}{l}\text { Counseling on the risks of having sex } \\
\text { before } 18 \text { years }\end{array}$} \\
\hline No & 954 & 92.7 \\
\hline Yes & 75 & 7.3 \\
\hline \multicolumn{3}{|l|}{ Maternity care $(n=752)$} \\
\hline No & 679 & 92.7 \\
\hline Yes & 55 & 7.3 \\
\hline \multicolumn{3}{|l|}{$\begin{array}{l}\text { Treatment for other gynecological } \\
\text { problems }(n=752)\end{array}$} \\
\hline No & 710 & 94.4 \\
\hline Yes & 42 & 5.6 \\
\hline \multicolumn{3}{|l|}{$\begin{array}{l}\text { Services related to experiences of sexual } \\
\text { violence }(n=752)\end{array}$} \\
\hline No & 738 & 98.1 \\
\hline Yes & 14 & 1.9 \\
\hline \multicolumn{3}{|l|}{ Other medical treatment* } \\
\hline No & 882 & 85.7 \\
\hline Yes & 147 & 14.3 \\
\hline
\end{tabular}

Note: *Treatment for malaria, diarrheal diseases, intestinal parasites, and injury.

\section{Level of Participant Responsiveness on Youth-Friendly Services}

Regarding the participant responsiveness fidelity dimension, $613(59.6 \%)$ get the YFS with a low level of participant responsiveness, $302(29.3 \%)$ get the YFS with a medium, and $114(11.1 \%)$, get the YFS with a high

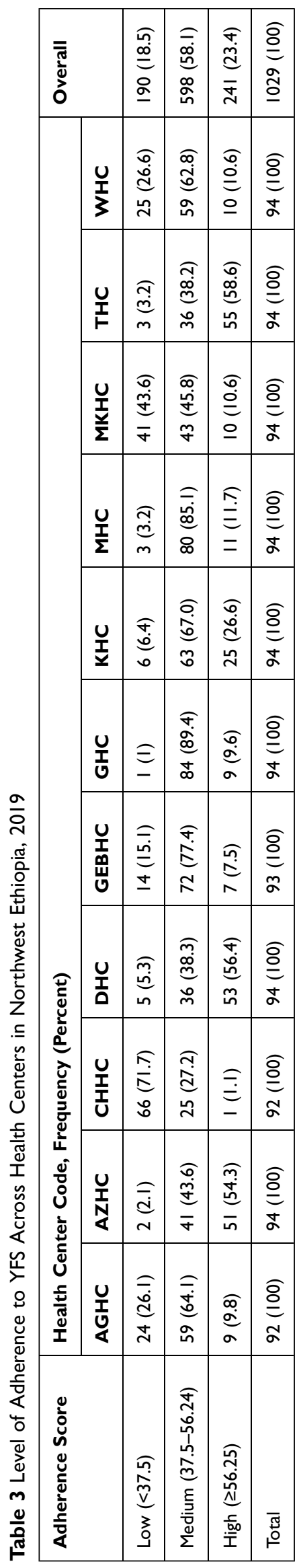




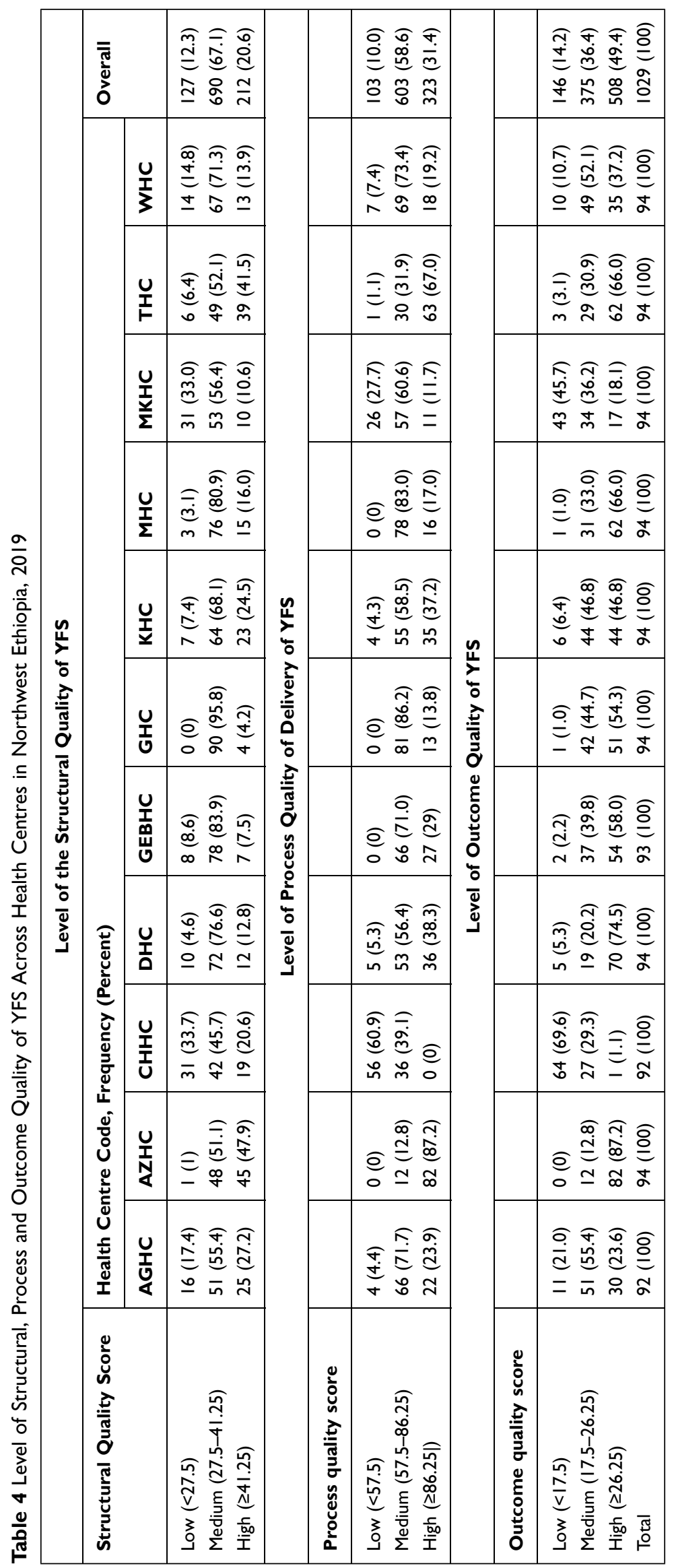


level of participant responsiveness. Tseda $\mathrm{HC}$ has the highest level of participant responsiveness 43 (45.7\%) followed by Kolladiba HC 28 (29.8\%) (Table 5).

\section{The Overall Level of Fidelity of Implementation of Youth-Friendly}

\section{Services}

The overall fidelity measure showed that only $48(4.7 \%$; $1.6-11.3 \%$ ) of youth received the YFS with a high level of implementation fidelity, followed by $770(74.8 \%, 57.6--$ $90.5 \%)$ with a medium and $211(20.5 \% ; 12.7-31.0 \%)$ with a low level of implementation fidelity. Tseda HC provided the YFS with a relatively higher level of FoI 19 (20.2\%), followed by Azezo HC 17 (18.1\%) (Table 6).

\section{Discussion}

This study assessed the level of implementation fidelity of YFS among youth attending YFS services in 11 public health centers found in Central Gondar Zone. The overall implementation fidelity of YFS was found to be low as compared to the WHO recommended implementation standard of YFS delivery. All the health centers have scored below the standard level of FoI of YFS.

The results showed that only 48 (4.7\%) of the respondents got the YFS with a high level of implementation fidelity. Besides, 770 (74.8\%) get the YFS with a medium, and 211 (20.5\%) get the YFS with a low level of fidelity of implementation.

This finding is lower than the WHO standard ${ }^{8}$ and the National Adolescent and Youth Health Strategy of Ethiopia, ${ }^{15}$ whereby the standards mentioned as the YFS should be delivered with a high level of implementation fidelity and quality. The possible explanations for the lower level of Fidelity of Implementation of YFS in the current study could be the nature of the YFS intervention, as it is a complex intervention and resource-intensive demanding trained providers and many other resources. Hence, resource limitation, lack of training of the YFS providers', budget constraint, and lack of NGOs support in the study area could be the potential reasons. ${ }^{40}$ This finding is also lower than a study conducted in Spain, where $57.2 \%$ of youth got the YFS with a high level of FoI. ${ }^{36}$ The possible reason for the low level of FoI of YFS in the current study as compared to the aforementioned study could be the difference in the health system infrastructure between the two countries. As Spain is a developed nation as compared to Ethiopia, the health

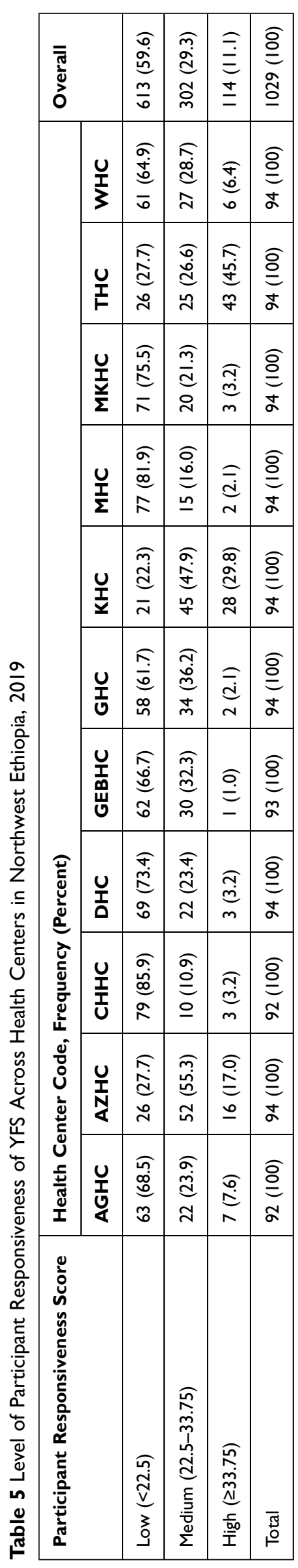




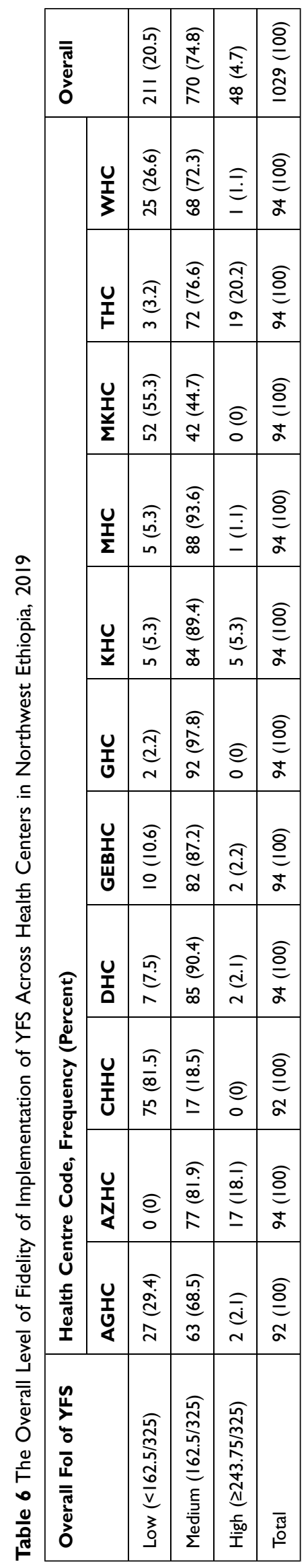

care system including the YFS program/provision may be stronger as compared to Ethiopia. The other possible justification could be the presence of some methodological differences in the dimensions used to measure FoI of YFS in the two studies. In the study conducted in Spain, the authors used dose, adherence and acceptance dimensions as a measure of fidelity, ${ }^{36}$ while in the current study adherence, quality of YFS care and participant responsiveness were used. Usually, the quality of care dimension is the very difficult part to be delivered with high fidelity, and hence the overall fidelity measure in the current study is lower. ${ }^{8}$

Regarding the adherence dimension, only $18.4 \%$ of youth get the YFS with a high level of adherence. This finding is lower than the $\mathrm{WHO}^{8}$ and the National Adolescent and Youth Health Strategy of Ethiopia. ${ }^{15}$ The possible explanation could be there could be a lack of commitment of the YFS providers in providing the YFS with a high level of adherence. Also, lack of training at all or lack of refresher training on YFS could be another possible reason for the provision of YFS with a low level of adherence. Usually, training motivates providers to deliver YFS with high adherence or fidelity. ${ }^{41}$ Moreover, many of the HCs may not have the YFS national standard manual in their clinic so that they may not read it and hence lack of adherence happens. Furthermore, lack of supportive supervision from the district, Zonal and Regional health offices may also be another justification for the low level of adherence in YFS provision.

The level of adherence is also lower as compared to a previous study conducted in Spain, where $30.4 \%$ of youth got the YFS with $100 \%$ adherence and $69.6 \%$ of the respondents got the YFS between $70 \%$ and $90 \%$ level of adherence. ${ }^{36}$ The possible justification could be the presence of disparities in the YFS setups including the presence and quality of training provided for health care providers in YFS provision between the countries.

Regarding the YFS quality of delivery fidelity dimension, only $12.3 \%$ in the structural dimension, $10 \%$ in the process dimension, and $14.2 \%$ in the outcome dimension get the services with a high level of quality. All of the YFS sites scored lower than the $\mathrm{WHO}^{8}$ and the National Adolescent and Youth Health Strategy ${ }^{15}$ standard (75\%) in the three of quality dimensions and the overall quality of YFS is categorized as "not good quality" or "below the standard." Besides, the quality of delivery score in this study is lower than a study conducted in Arbaminch; 
whereby the overall quality of YFS delivery score input, process, and youth clients' satisfaction was $54.41 \%$, $42.0 \%$, and $49.1 \%$, respectively. ${ }^{30}$ The possible reason for the lower findings of the current study as compared to a study conducted in Arbamnich could be the presence of variation in the study settings between the two studies conducted. The aforementioned study was conducted only in urban settings whereas in the current study both urban and rural HCs were considered. ${ }^{30}$ Usually, in the urban settings, the YFS clinic gets better support from many stakeholders, NGOs and even the Regional and Zonal health offices. Likewise, in urban HCs there are more health care providers who are trained and experienced in providing YFS as compared to rural HCs. ${ }^{10}$

Furthermore, the YFS quality of delivery measure is still lower as compared to a study conducted in Mexico $(17.5 \%) .^{42}$ The possible explanation for the lower quality score in the current study as compared to Mexico's study could be the presence of difference in the socio-economic, the overall health care system and the YFS program competency between the two countries. ${ }^{16,42}$

The participant responsiveness dimension of this study showed that only $59.5 \%$ of youth got the YFS with a high level of fidelity. This finding is against the WHO standard and the National Adolescent and Youth Health Strategy of Ethiopia, whereby full youth participation and involvement in the YFS delivery is a milestone in YFS delivery. ${ }^{8,15}$ The possible justification for the low level of participant responsiveness in the current study could be, since the study included rural HCs, in most of the HCs youth may not participate in the design, planning and as a peer counselor in the YFS provision. Further, there could be poor youth and community perception for YFS in the study area that may lower youth involvement in YFS. This finding implies that participating youth in the YFS program design and service provision is crucial to deliver the YFS with high fidelity. Therefore, programmers, implementers, strategists and policymakers better consider youth involvement in the YFS program. Further research has to be conducted to explore the barriers to the implementation fidelity of youth-friendly services.

The findings of the study implied that rigorous efforts to build systems that apply the policy and principles of implementation fidelity of YFS in the health facilities are vital. These initiatives will not only benefit youth but the health system overall, as the principles for implementation fidelity of the National Adolescent and Youth Health Strategy are in step with those of YFS to be delivered with a high level of adherence, quality of delivery and youth participation. ${ }^{15}$ Another implication of the study finding is considering high levels of implementation fidelity of YFS is compulsory to avert the SRH problems among youth, in addition to the YFS service expansion. Furthermore, investing in effective interventions (like YFS), is important to improve its implementation fidelity. There is a need to strengthen the YFS to be delivered with a high level of fidelity to achieve the desired intervention outcomes within a short period of time.

\section{Limitations}

As a limitation of this study, the measure of Fidelity of implementation of YFS was from youth perspectives, that means, the study did not consider the providers' perspective and direct observation. Also, the study did not explore barriers to the implementation fidelity of YFS qualitatively.

\section{Conclusion}

In this study, the level of implementation fidelity of YFS is found to be low in all three dimensions of fidelity. The findings showed a discrepancy between the implementation policy and the actual/practical health facility level performance. Previous studies only investigated the proportion of YFS service utilization among youth, without considering its implementation fidelity. Hence, this study generates new evidence by showing the actual program-level performance on the implementation fidelity of YFS which has a better input to strengthen the YFS intervention.

Rigorous efforts to build systems that apply the policy and principles of implementation fidelity of YFS in the health facilities are recommended. Therefore, the government, partners, programmers and implementers shall give primer attention on improving the implementation fidelity of YFS. Further studies that can address the barriers to the implementation fidelity of Youth-Friendly Services are recommended. Finally, the authors' recommend to conduct studies that will identify the factors that contribute to the fidelity of YFS by employing advanced statistical regression models.

\section{Abbreviations}

AIDS, acquired immune deficiency syndrome; CI, confidence intervals; FMoH, Federal Ministry of Health; FoI, Fidelity of Implementation; HC, health center; HIV, human immune deficient virus; NGOs, non-governmental organizations; SRH, sexual and reproductive health; STIs, sexually transmitted infections; VCT, Voluntary Counseling and 
Testing; WHO, World Health Organization; YFS, youthfriendly services.

\section{Data Sharing Statement}

All the raw data supporting the findings can be obtained from the corresponding author upon reasonable request.

\section{Ethics Approval and Informed Consent}

Ethical clearance was obtained from the University of Gondar Institutional Review Board (IRB) with reference number, R. no.-O/V/P/RCS/05/1047/2019. Then, official permission letter was cascade from respective Zonal to local authorities including Central Gondar Zonal health offices, Gondar City Administration Health Department and the 11 districts health offices including the $11 \mathrm{HCs}$ During data collection time, informed and written consent was obtained from each study participant. Further, for those participants below 18 years, individual assent and written informed consent were obtained from a parent or guardian. Furthermore, confidentiality was maintained through anonymity and privacy measures to protect respondent's right through the research process. This study was conducted in accordance with the Declaration of Helsinki.

\section{Acknowledgments}

The authors acknowledged Central Gondar Zone Health Department officials for their support. Besides, administrators of each district health offices and head of HCs, the data collectors, the supervisors, the YFS providers and the respondents are also acknowledged.

\section{Author Contributions}

All authors made a significant contribution to the work reported, whether that is in the conception, study design, execution, acquisition of data, analysis and interpretation, or in all these areas; took part in drafting, revising or critically reviewing the article; gave final approval of the version to be published; have agreed on the journal to which the article has been submitted; and agree to be accountable for all aspects of the work.

\section{Funding}

University of Gondar covered the budget for data collection, but had no technical role in the research.

\section{Disclosure}

The authors declare that they have no competing interests.

\section{References}

1. Angel WA Resolutions on Youth Rights Adopted by the General Assembly and Economic and Social Council of the United Nations: 1965-1993. The International Law of Youth Rights. Brill Nijhoff; 2015 139-290.

2. Secretariat C Global youth development index and report 2016: commonwealth secretariat; 2016.

3. UNICEF. The state of the world's children 2011-executive summary: adolescence an age of opportunity: Unicef; 2011.

4. DESA U. Youth population trends and sustainable development. Population facts 2015; 2015;1:1-4.

5. CSA. The 2007 population and housing census of Ethiopia. Statistical Summary Report at National Level. Addis Ababa, Ethiopia: Central Statistical Agency; 2008:7-60.

6. Bearinger LH, Sieving RE, Ferguson J, Sharma V. Global perspectives on the sexual and reproductive health of adolescents: patterns, prevention, and potential. Lancet. 2007;369(9568):1220-1231. doi:10.1016/S0140-6736(07)60367-5

7. Gore FM, Bloem PJ, Patton GC, et al. Global burden of disease in young people aged 10-24 years: a systematic analysis. Lancet. 2011;377(9783):2093-2102. doi:10.1016/S0140-6736(11)60512-6

8. WHO. Quality assessment guidebook: a guide to assessing health services for adolescent clients; 2009.

9. WHO. Adolescent friendly health services: an agenda for change. World Health Organization; 2003.

10. WHO. Global consultation on adolescent friendly health services-a consensus statement. Geneva: World Health Organization; 2002:29.

11. Mmari K, Sabherwal S. A review of risk and protective factors for adolescent sexual and reproductive health in developing countries: an update. $J$ Adolesc Health. 2013;53(5):562-572. doi:10.1016/j. jadohealth.2013.07.018

12. WHO. Quality of care: a process for making strategic choices in health systems. World Health Organization; 2006.

13. WHO. External evaluation 2008-2012: advancing sexual and reproductive health. World Health Organization; 2013.

14. WHO. Orientation programme on adolescent health for health care providers; 2006.

15. Oljira L National Adolescent and Youth Health Strategy (2016-2020) Federal Democratic Republic of Ethiopia Ministry of Health; 2016.

16. FDRE. MOH. Standards on Youth Friendly Reproductive health Services service delivery guideline \& Minimum service delivery package on YFRH services; 2006:1-48.

17. Sawyer SM, Patton GC Platforms for delivery of adolescent-friendly health care. Health and Nutrition in Adolescents and Young Women: preparing for the Next Generation. Karger Publishers; 2015:71-85.

18. WHO. Global accelerated action for the health of adolescents (AA-HA!): guidance to support country implementation: summary. Global accelerated action for the health of adolescents (AA-HA!). Guidance to support country implementation: summary; 2017.

19. WHO. Adolescent pregnancy. Fact sheet No. 364. September 2014; 2014:1-4.

20. LIVE WAW. Facts on the sexual and reproductive health of adolescent women in the developing world; 2010:1-4.

21. Patton GC, Bowes G, Sawyer SM, Homel R, Stanley FJ. Towards a national agenda for youth? Med J Aust. 2005;183(8):394-395. doi:10.5694/j.1326-5377.2005.tb07098.x

22. Patton GC, Coffey C, Cappa C, et al. Health of the world's adolescents: a synthesis of internationally comparable data. Lancet. 2012;379(9826):1665-1675. doi:10.1016/S0140-6736(12)60203-7 
23. Durlak JA. Studying program implementation is not easy but it is essential. Prev Sci. 2015;16(8):1123-1127. doi:10.1007/s11121-0150606-3

24. Durlak JA, DuPre EP. Implementation matters: a review of research on the influence of implementation on program outcomes and the factors affecting implementation. Am J Community Psychol. 2008;41 (3-4):327. doi:10.1007/s10464-008-9165-0

25. Berhane F, Berhane Y, Fantahun M. Adolescents' health service utilization pattern and preferences: consultation for reproductive health problems and mental stress are less likely. Ethiop J Health Dev. 2005;19(1):29-36. doi:10.4314/ejhd.v19i1.9968

26. Dagnew T, Tessema F, Hiko D. Health service utilization and reported satisfaction among adolescents in Dejen District, Ethiopia: a cross-sectional study. Ethiop J Health Sci. 2015;25(1):17-28. doi:10.4314/ejhs.v25i1.4

27. Jaleta A, Assefa A, Amentie M. Youth friendly reproductive health service utilization and associated factors among youths in Metekel Zone, North West Ethiopia. Int J Ophthalmol Vis Sci. 2017;2 (2):59-67.

28. Iyer P, Clarke D, Aggleton P Barriers to HIV and sexuality education in Asia. Health Education; 2014:1-5.

29. Pandey PL, Seale H, Razee H. Exploring the factors impacting on access and acceptance of sexual and reproductive health services provided by adolescent-friendly health services in Nepal. PLoS One. 2019;14(8):e0220855. doi:10.1371/journal.pone.0220855

30. Mulugeta B, Girma M, Kejela G, Meskel FG, Andarge E, Zerihun E. Assessment of youth-friendly service quality and associated factors at public health facilities in southern Ethiopia: a facility-based cross-sectional study. Biomed Res Int. 2019;2019:1-9. doi:10.1155/ 2019/9696278

31. Renju J, Andrew B, Nyalali K, et al. A process evaluation of the scale up of a youth-friendly health services initiative in northern Tanzania. J Int AIDS Soc. 2010;13(1):32. doi:10.1186/1758-2652-13-32

32. Geary RS, Webb EL, Clarke L, Norris SA. Evaluating youth-friendly health services: young people's perspectives from a simulated client study in urban South Africa. Glob Health Action. 2015;8(1):26080. doi:10.3402/gha.v8.26080
33. Domitrovich CE, Greenberg MT. The study of implementation: current findings from effective programs that prevent mental disorders in school-aged children. J Educ Psychol Consult. 2000;11(2):193-221. doi:10.1207/S1532768XJEPC1102 04

34. Dusenbury L, Brannigan R, Falco M, Hansen WB. A review of research on fidelity of implementation: implications for drug abuse prevention in school settings. Health Educ Res. 2003;18(2):237-256. doi:10.1093/her/18.2.237

35. Elliott DS, Mihalic S. Issues in disseminating and replicating effective prevention programs. Prev Sci. 2004;5(1):47-53. doi:10.1023/B: PREV.0000013981.28071.52

36. Escribano S, Espada JP, Orgilés M, Morales A. Implementation fidelity for promoting the effectiveness of an adolescent sexual health program. Eval Program Plann. 2016;59:81-87. doi:10.1016/j. evalprogplan.2016.08.008

37. Rogers E. Diffusion of Innovation. Fifth ed. New York NY: Free Press; 2003.

38. CGZA. Population and urban planning report. Central Gondar Zone Administration for 2018/2019 budget year Gondar, Ethiopia 2018/ 2019; 2018/2019.

39. Kotrlik J, Higgins C. Organizational research: determining appropriate sample size in survey research appropriate sample size in survey research. Inf Technol Learn Perform J. 2001;19(1):43.

40. Fikree FF, Zerihun H. Scaling up a strengthened youth-friendly service delivery model to include long-acting reversible contraceptives in Ethiopia: a mixed methods retrospective assessment. Int $J$ Health Policy Manag. 2020;9(2):53. doi:10.15171/ ijhpm.2019.76

41. Nurjono M, Shrestha P, Ang IYH, et al. Implementation fidelity of a strategy to integrate service delivery: learnings from a transitional care program for individuals with complex needs in Singapore. $B M C$ Health Serv Res. 2019;19(1):177. doi:10.1186/s12913-019-3980-x

42. Villalobos A, Allen-Leigh B, Salazar-Alberto J, et al. Quality of reproductive healthcare for adolescents: a nationally representative survey of providers in Mexico. PLoS One. 2017;12(3):e0173342. doi:10.1371/journal.pone. 0173342
Risk Management and Healthcare Policy

\section{Publish your work in this journal}

Risk Management and Healthcare Policy is an international, peerreviewed, open access journal focusing on all aspects of public health, policy, and preventative measures to promote good health and improve morbidity and mortality in the population. The journal welcomes submitted papers covering original research, basic science, clinical \& epidemiological studies, reviews and evaluations,

\section{Dovepress}

guidelines, expert opinion and commentary, case reports and extended reports. The manuscript management system is completely online and includes a very quick and fair peer-review system, which is all easy to use. Visit http://www.dovepress.com/testimonials.php to read real quotes from published authors. 